\title{
A New Profile Measurement Method for Thin Film Surface
}

\author{
ShuJie Liu ${ }^{1, *}$, Yuan L. Zhang ${ }^{1}$ and Hong C. Zhang ${ }^{1,2}$ \\ ${ }^{1}$ School of Mechanical Engineering Dalian University of Technology Dalian, China, 116024 \\ ${ }^{2}$ Department of Industrial Engineering, Texas Tech University, Lubbock, USA, TX 79409
}

\begin{abstract}
In order to measure the surface profile of the thin films in wide area with high accuracy, the research on the new measurement method was conducted and many patents on measurement method and system were researched. Based on the physical properties of soft thin film, the method of using white interferometer and ball cantilevers for measuring the surface profile of thin film is proposed. Then, the multi-ball-cantilever AFM system using a multi-ball-cantilever is developed that had 8 cantilevers in $2 \mathrm{~mm}$ and each cantilever had the ball stylus with diameter of $10.9 \mu \mathrm{m}$. Using this system, the measurement experiments on the step and transparent material was conducted. The results on the step that the precision of the system's measuring error is less than $2 \%$ and the measuring standard deviation is less than $1 \mathrm{~nm}$. The result on the step indicated that it is possible to measure the surface with high accuracy. The result on the transparent material showed the feasibility for measuring the thin film by this developed system.
\end{abstract}

Keywords: Multi-ball cantilever, surface profile measurement, thin film, white light interferometer.

\section{INTRODUCTION}

The development of the semiconductor industry is moving towards miniaturization and high integration. Semiconductor industry with high-performance and low-cost is the motivation of the development of information technology. Lithography plays an extremely important role in semiconductor integration process. The short-wavelength light irradiation method is usually used to improve the resolution, but the impact on the exposure is increasing because of the photoresist surface irregularity, which is due to the shallow depth of focus if the wavelength is reduced. In traditional lithography, the surface profile of photoresist film does not need to be measured, and only the exposure in a required range of accuracy needs to be controlled through correcting the measurement system calibration and the measurement systems focus. But as the semiconductor technology moving toward a more accurate direction, the photoresist surface irregularity has a significant impact on the exposure's accuracy even when the difference of surface unevenness is only several $10 \mathrm{~nm}$. Therefore, the surface profile measurement of the photoresist and the corresponding measurement methods are key issues to be studied in the semiconductor industry [13].

Now, surface profile measurement methods are mainly divided into two categories. One is non-contact measurement technique by use of light exposure. The other is contact measurement technique by contacting with the surface of the specimen. Non-contact measurement technique is to use the light irradiation to measure the surface of the specimen, without forces acting on it and this method belongs to the non-invasive measurement [4]. And there is no deformation in the process of measuring. The instruments, such as white light interferometer and confocal microscope, are widely used in the wide-area measurement. Since there is no contact scanning in the measurement, the non-contact measurement has high-speed. In the measurement accuracy, the noncontact measurement can get high accuracy in the vertical by the phase and intensity of light, but the lateral resolution, depending on the half-wavelength of light, is lower than the vertical resolution. Meanwhile, the experimental material exposed to the light waves cannot receive the reflected light signals due to the improper angles of the optical axis, which could not be measured. Because of the multiple reflections of the light, the correct profile of the translucent film couldn't be measured. When the thickness of a film is only several hundred $\mathrm{nm}$, which is approximately several times the wavelength of light, the reflected lights transmitted from the film and from the bottom surface will interfere, and if they are considered as the reflected light to resolve the relationship of the phase and the height of light, the correct profile cannot be observed. However, this problem will not be obvious when the film has sufficient thickness. Contact measurement is with a high reliability, especially in the vertical, and $1 \mathrm{~nm}$ can be measured. The measurement accuracy in the lateral can be improved by selecting the appropriate contact. However, the weakness of contact measurement is that the contact force may cause deformation of the specimen surface. Moreover, the time cost in a wide area measurement is relatively increased due to the contact with the surface. Atomic force microscope (AFM) and surface roughness measuring gauge are some typical examples of contact measuring instrument $[5,6]$.

We study some patents on measurement methods and systems. Patent US2014020140 [7], titled "multi-head probe 


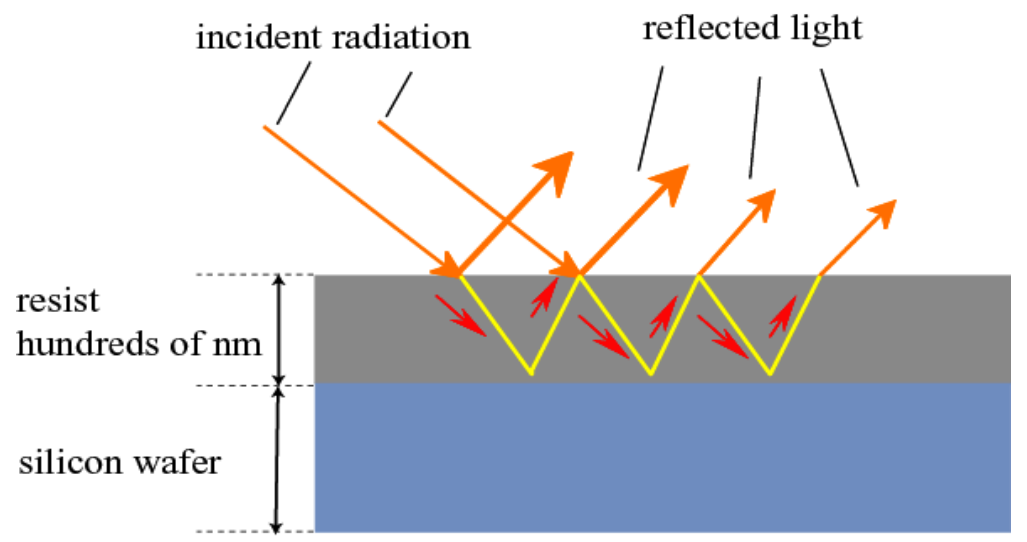

Fig. (1). Multiple interferences phenomenon in the film.

and scanning method thereof" provides a multi-head probe suitable for an atomic force microscopy (AFM) comprising of a tip base, a single cantilever beam and at least two tips. The tip base has a tip end, which is grounded to form a surface. The cantilever beam is connected to the tip base and for supporting the tip base. Patent CN102257353 [8] titled "device and method for the three-dimensional optical measurement of strongly reflective or transparent objects" relates to a device for three-dimensionally measuring an object, comprising of a first projection device, having a first infrared light source for projecting a displaceable first pattern onto the object, and at least one image recording device for recording images of the object in an infrared spectral range. Patent US20120218560 [9], titled "method and apparatus for performing film thickness measurement using white light scanning interferometry" relates to a method and an apparatus for measuring the thickness of a transparent film by broad band interferometry, comprising of the steps of preparing a correlogram of the film by an interferometer, applying a Fourier transformation to the said correlogram to obtain a Fourier phase function, removing a linear component thereof by applying a second integral transformation to the remaining non-linear components to obtain an integral amplitude function of the said non-linear component. Identifying the peak location of said integral amplitude function and determining the thickness of the film as the double value of the abscissa at said peak location and considering a refractive index of a film which is dependent on wavelength. Patent WO2014024614 [10], titled "optical measurement device and optical measurement system" provides an optical measurement device and an optical measurement system whereby test subject information and measurement data can be reliably correlated with each other. Patent US20140025336 [11], titled "shape measuring apparatus and control method of shape measuring apparatus", is a control method of a shape measuring apparatus which divides a curve indicating a movement path of a probe into a plurality of sections.

\section{SURFACE PROFILE MEASUREMENT METHOD OF THIN FILM}

\subsection{The Thin Film Properties}

In this study, the measurement object (photoresist) has the following characteristics: (1). the film thickness of sev- eral hundred $\mathrm{nm} ;(2)$. the surface shape changes mitigation, the undulation within $0.2 \mathrm{~mm}-20 \mathrm{~mm}$ and the amplitude of several tens of nm; (3). "soft" material, the experimental elastic modulus is about $30 \mathrm{GPa}$, which is $1 / 7$ times of silicon's; (4). transparent or translucent.

The problem of non-contact measurement is due to the translucency of the film and its thickness, and the thickness of photoresist is about several hundred $\mathrm{nm}$, which easily leads to the phenomenon of interference as show in Fig. (1). Thus, it is difficult to measure the thin film using white light interferometer or confocal microscopy. Using contact measuring method will require a lot of time to measure a large surface area profile of photoresist coated on the wafer. The surface wave level of the resist film measured is several hundred microns, and the difference of the height is $10 \mathrm{~nm}$. In order to get the shape of the resist film, high-precision measurements in the vertical is needed while the lateral resolution does not have high requirements, and some special high-frequency component does not need to be detected. Therefore, the advantages of contact measurement with high lateral resolution are negated [12].

What's worth noting is that by optical measurement method it is difficult to realize the high accuracy measurement of the thin-film in principle, and contact measurement can obtain relatively accurate results if the force effects do not cause defects on the surface for the resist film, even though it requires considerable time [13]. If the advantages of the optical measurement (high speed and without deformation) are used, the problems we faced can be solved. Thus, the surface profile measurement technique of the thin films combining optical and mechanical measurement is proposed [14].

\subsection{The Principle of the Measuring Method}

The surface profile measurement method proposed above is expressed in Fig. (2). The main characteristics are showed in the following:

(A) combining optical measurement (white light interferometer) and mechanical contact (multi ball cantilever)

The fundamental of the proposed measurement method is showed in Fig. (3). The surface profile of the thin film can be obtained indirectly without multiple reflections by optical measurement instrument and the medium. 


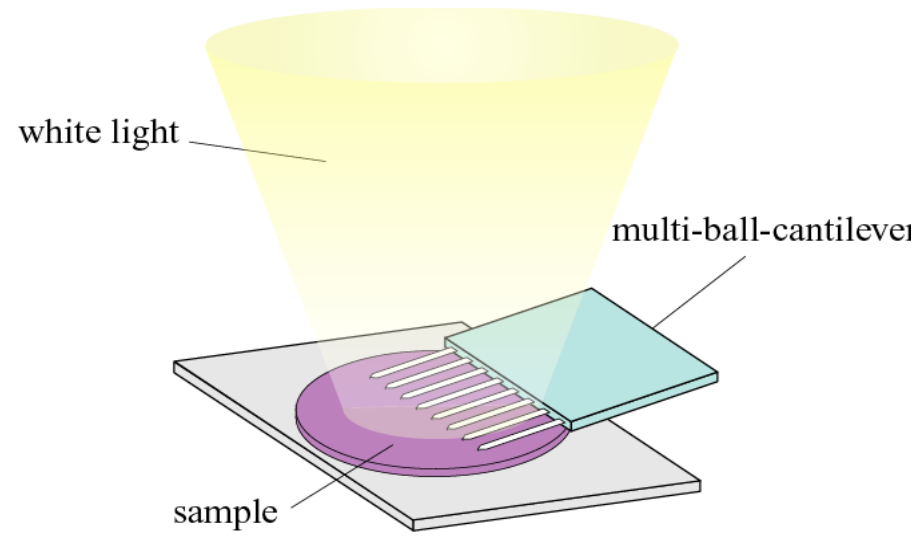

Fig. (2). Single-phase full bridge inverter.

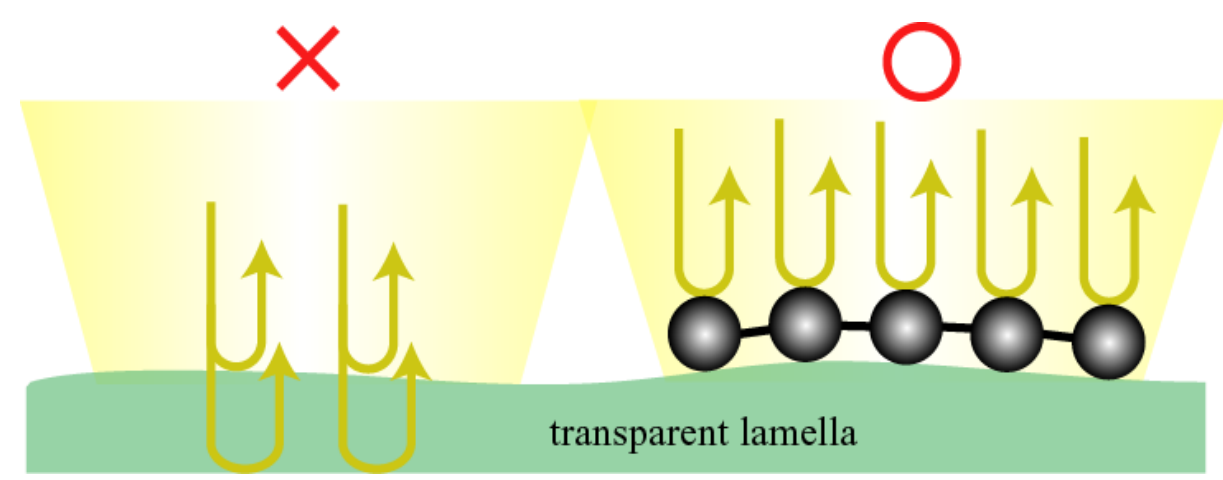

Fig. (3). The fundamental of the proposed measurement method.
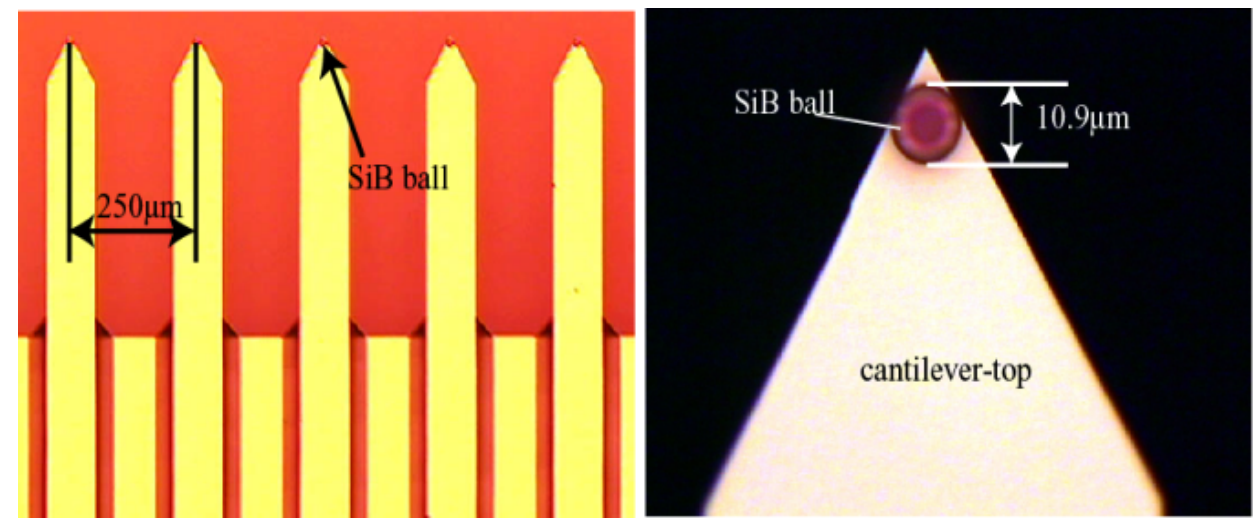

Fig. (4). A linear array of spherical probes.

(B) multiple spherical probes are used in the contact part

The probes are spherical, which can reduce the effect of the contact on the film, and multiple probes are used to achieve wide-area measurements. A linear array of spherical probes is used in the measurement, as shown in Fig. (4). To avoid interference, optical measurement is used to measure the upper surface of scanning probe. To reduce the impact of the contact on shapes, spherical probes with large diameter are adopted.

(C) The application of the white interferometer in optical measurement part

The application of the white interferometer can realize large area scanning measurement. In the study, the New
View's white light interferometer is used as optical measuring instruments. Three-dimensional geometry of the highprecision surface is detected by the white light interference principle. Surface roughness, waviness, roughness, step height, etc. can be qualitative and quantitative measured through flotation worktable, which has the function of stitching and micro-geometry error analysis. The concrete aspects are demonstrated as following: (1). the measurement range in the vertical is $1 \mathrm{~nm}-15000 \mu \mathrm{m},(2)$. the reproducibility of the step height standard is less than $0.5 \%$, (3). the measurement error of the step height standard is less than $0.75 \%$, (4). the reproducibility of RMS is $0.1 \mathrm{~nm}$.

A multi-ball cantilever measuring device was constructed as shown in Fig. (5). The multi-ball cantilever is NANO- 


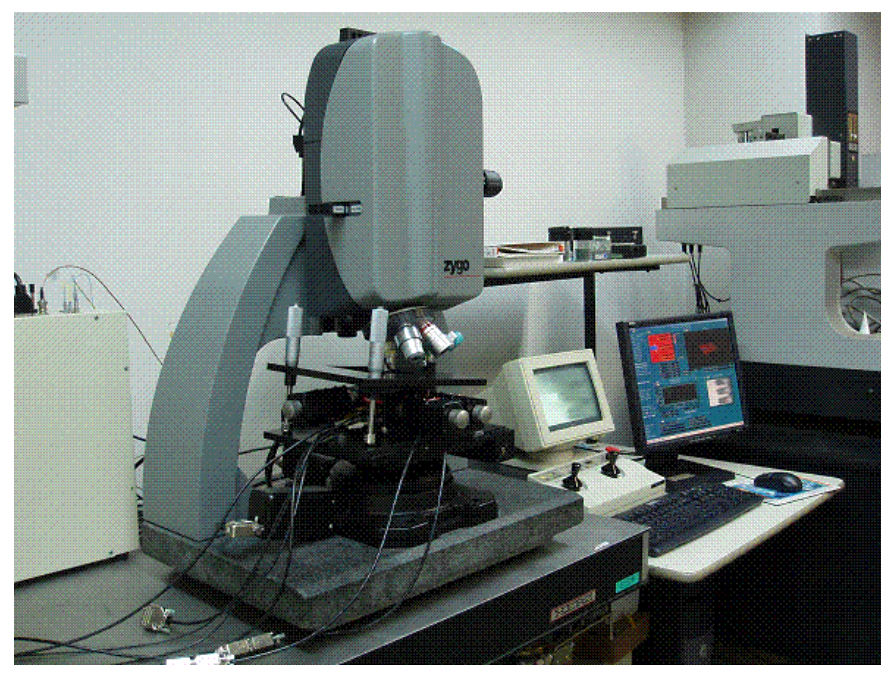

Fig. (5). The multi-ball cantilever measurement system.

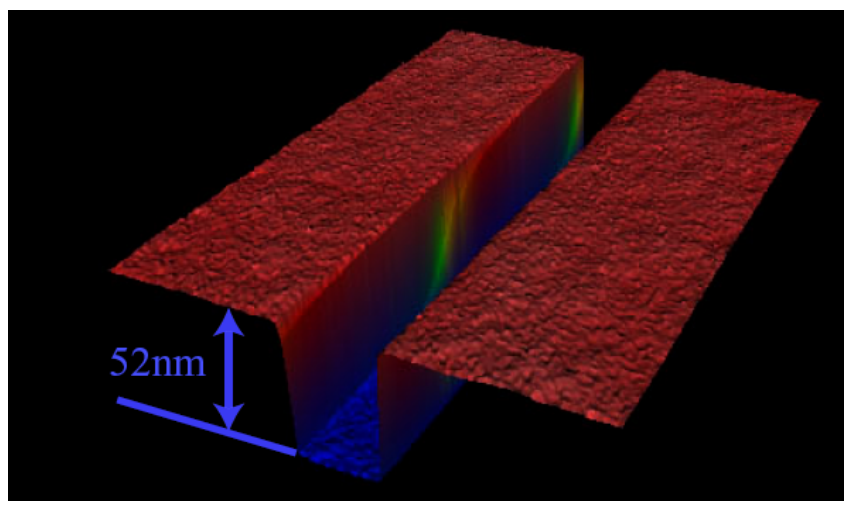

Fig. (6). A step height standard.

WORLD Arrow TL8-50 which uses 8 cantilevers spaced 250 $\mu \mathrm{m}$ apart, and each cantilever holds a ball stylus $10.9 \mu \mathrm{m}$ in diameter.

The white interferometer used for the experimental studies is a ZYGO NewView5000. Using the interference strength detection method, New View 5000 can detect height information with a height resolution of $0.1 \mathrm{~nm}$ and profile heights ranging from $1 \mathrm{~nm}$ to $15000 \mu \mathrm{m}$.The measuring performance of the instrument is examined by the following basic experiments [15].

\section{MEASUREMENT EXPERIMENT}

\subsection{Height Standard}

A step height standard that is a nominal $52 \mathrm{~nm}$ step in height and $100 \mu \mathrm{m}$ in width is measured in the experiment, as shown in Fig. (6). In this experiment, three cantilevers are used to simplify the evaluation. Step portion and both side flat portions are measured using three neighboring ball cantilevers with $250 \mu \mathrm{m}$ spacing. In order to calibrate the variation in the diameter of the ball on each probe, and the thickness of the cantilever and the mounting position of the cantilever, the sample plane was measured. The measurement was performed in ten different points. In measurement, there is a height difference between the middle cantilever and the cen- ter of the either side cantilever, the step value is calculated from this difference. Here, this calculation method is called "three-point relative method". Fig. (7) shows the measurement image in the experiment from white light interferometer. Processing the data and the measure results are gained in Table 1. The measurement error is less than $2 \%$ and the standard deviation is $0.9 \mathrm{~nm}$. The experiments achieve the purpose of high-accuracy measurements.

Table 1. Result of the step height standard measurement.

\begin{tabular}{|c|c|}
\hline The Times of Measurement & The Height (nm) \\
\hline \hline 1 & 50.8 \\
\hline 2 & 51.6 \\
\hline 3 & 50.7 \\
\hline 4 & 51.5 \\
\hline 5 & 52.5 \\
\hline 6 & 49.8 \\
\hline The mean & 51.2 \\
\hline The standard deviation & 0.9 \\
\hline
\end{tabular}




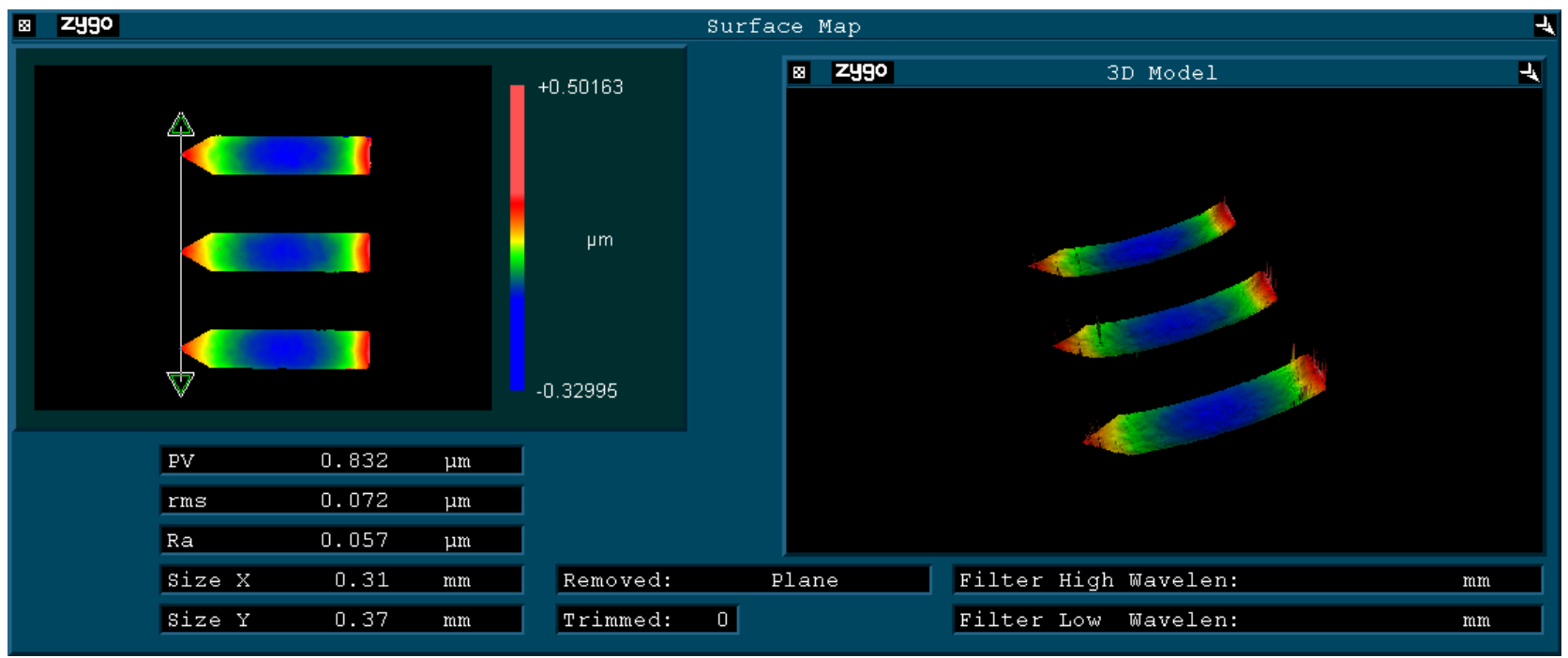

Fig. (7). Measurement image using three ball cantilevers.

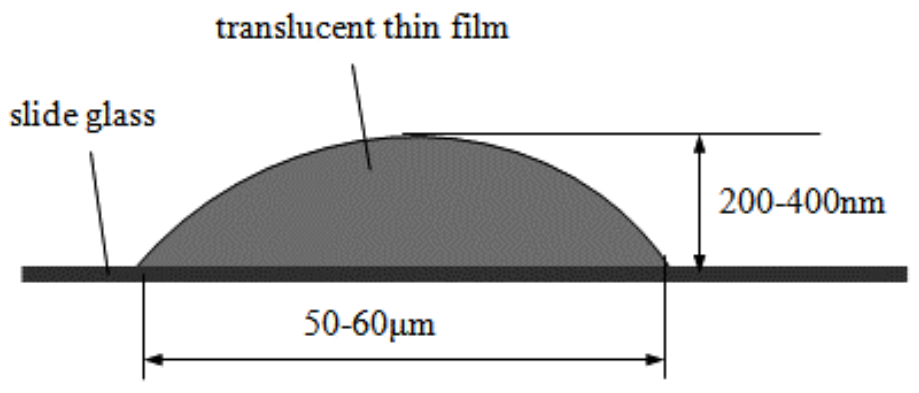

Fig. (8). Transparent resin sample.

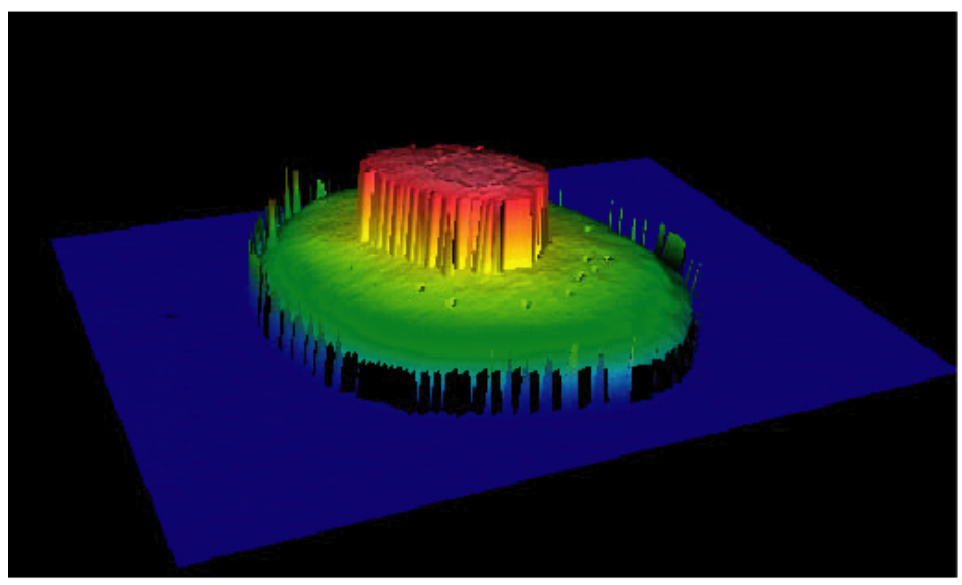

Fig. (9). The measurement results by white light interferometer, Profile cannot be measured correctly by white light interferometer because of multiple interference.

\subsection{Measurement of Step Height Standard}

In order to confirm whether it is possible to measure transparent thin films using the developed apparatus, it is necessary to compare with the actual value. This experiment uses the resin film with the thickness of 200-400 $\mathrm{nm}$ and the diameter 50-60 $\mu \mathrm{m}$, as shown in Fig. (8). The white interferometer measuring causes multiple reflections and the discontinuous measurement profile are obtained as in Fig. (9).

The resin film sample used in this experiment is not the reference sample with real value. Therefore, in order to 


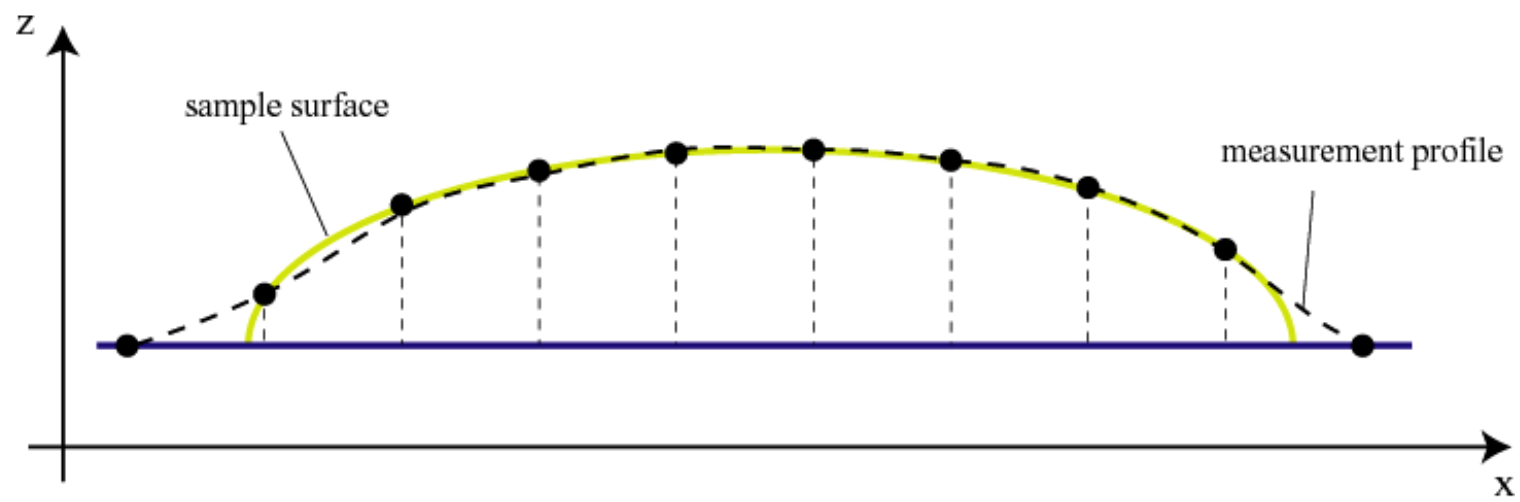

Fig. (10). Measurement method.

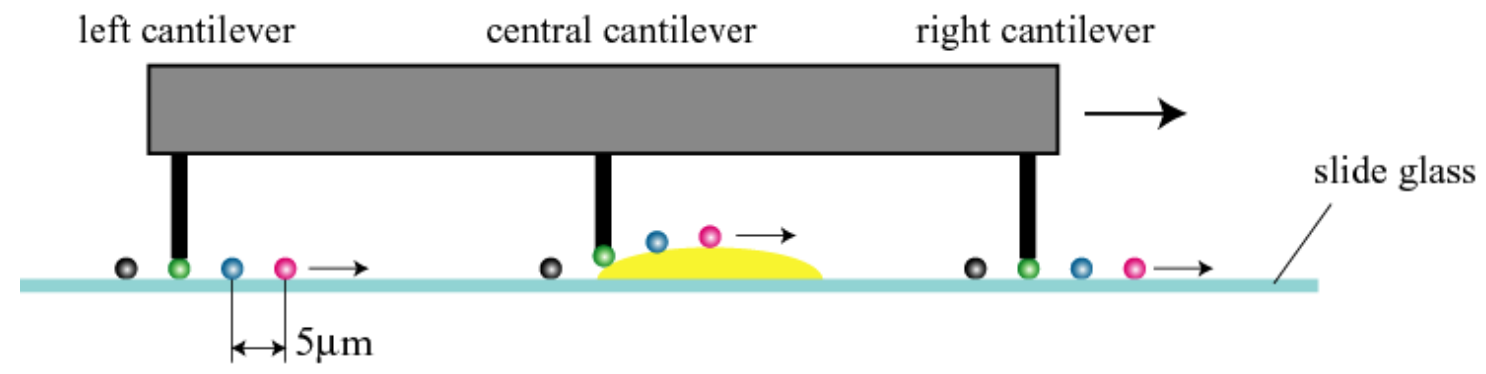

Fig. (11). Measurement process.

verify the measurement result, the sample surface is measured when it was with and without gold evaporation. Thickness of the gold deposition is $25-30 \mathrm{~nm}$. When gold is deposited on the film, the surface becomes reflective and does not let any light pass through; making measurement in the white light interferometer possible. For film samples used being 50-60 $\mu \mathrm{m}$ in diameter, one cantilever only will be in contact with the sample because multi-ball cantilever has $250 \mu \mathrm{m}$ intervals. Therefore, it is possible to apply the threepoint relative method here. It is decided to measure the height of each portion of the thin film in this measurement method. The measurement method and measurement process are shown in Fig. (10) and Fig. (11) respectively.

We compare the profile by the developed apparatus and by white light interferometer. The measurement results based on four conditions (the presence or absence of gold deposition and using developed multi-cantilever apparatus or only white light interferometer) were shown in Fig. (12).

A good measurement result is not possible for the effect of multiple reflections in Fig. (12) (a). Comparing Fig. (12) (b), (c), and (d), we have similar results. Focusing on the measurement result $b$, measurement is performed by the developed device on the sample without coating, since it is by the mechanical contact; it is not encounter the effects of multiple reflections. The result shapes can be considered which are not so different in the Fig. (12) (b) and (d), and there is little change in shape due to the gold deposition. Experiments show that the developed device may overcome the effect of the multiple reflections, and the surface profile of the film can be measured.

\section{CURRENT \& FUTURE DEVELOPMENTS}

In order to realize fast, highly accurate measurement of the surface profile of photoresist, the paper proposed a measuring method combing optical measurement and mechanical contact and a multi-ball cantilever AFM system is constructed for the task. The experimental apparatus is evaluated by the basic experiments on the step height standard and the surface profile measurement on the translucent film. In the experiment of step height standard, the results are observed that the measurement error is less than $2 \%$ and the standard deviation is within $0.9 \mathrm{~nm}$, which proved the feasibility of using this device to measure the step height standard with high accuracy. Then, the translucent thin film is measured by the proposed system, which demonstrated that this system can be used in measurement of the soft thin film, where the white light interferometer couldn't work. The result on the transparent material showed the feasibility of measuring the thin film by this developed system.

In future, a study will be conducted on the accuracy of the scanning measuring methods. Patent TW201346213 [16], titled "Calibration of an optical metrology system for critical dimension application matching" presents methods and systems for matching critical dimension measurement applications at high precision across multiple optical metrology systems. Calibration of multiple probes should be the point in our measurement system. Now the device is using one-dimensional probe on a straight line, in the future, 2dementional set probes' measurement method will also be studied. 


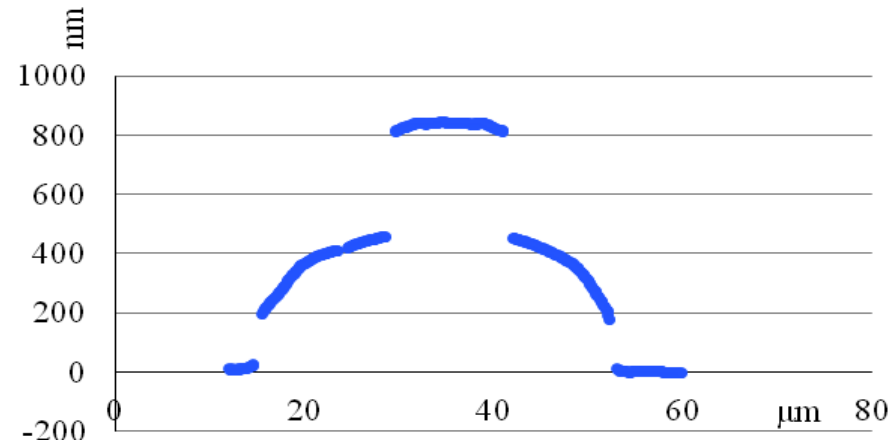

(a) Sample without coating is measured by white light interferometer.

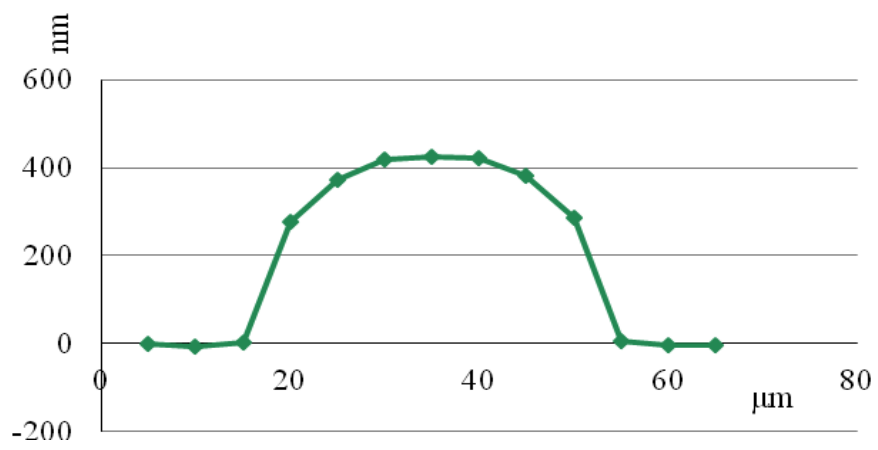

(b) Sample without coating is measured by developed instrument.

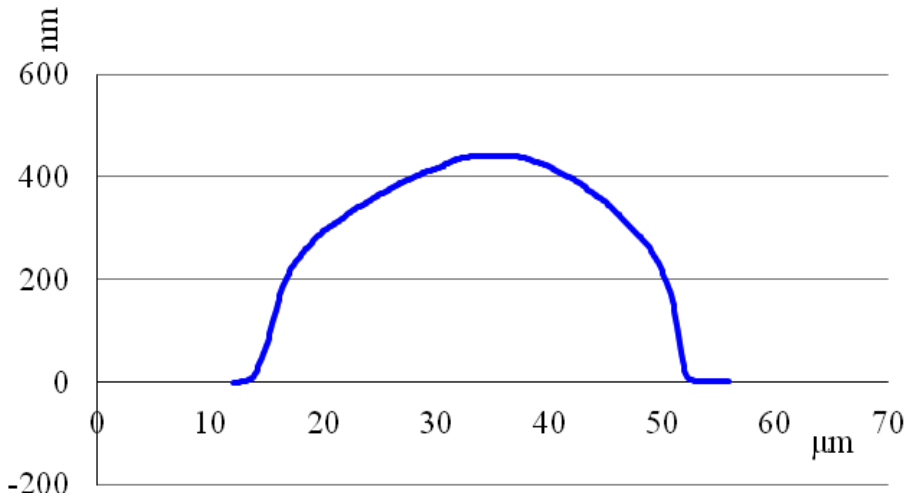

(c) Sample with coating is measured by white light interferometer.

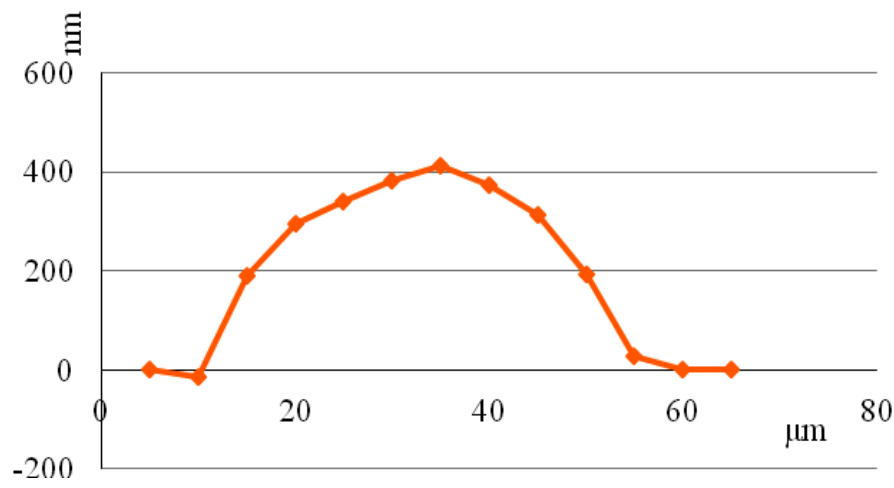

(d) Sample with coating is measured by developed instrument.

Fig. (12). Measurement results. 


\section{CONFLICT OF INTEREST}

The authors confirm that this article content has no conflict of interest.

\section{ACKNOWLEDGEMENTS}

This work is jointly supported by the National Natural Science Fund of China (No. 51205043) and Specialized Research Fund for the Doctoral Program of Higher Education (No. 20120041120033).

\section{REFERENCES}

[1] ITRS. International Technology Roadmap for Semiconductor (ITRS 2009), 2010.

[2] W. Conley, and J. Bendik, "Is ArF the final wavelength?" Proceeding SPIE, 2004, 5376, pp. 16-20.

[3] Z. X. Wu, and Y Q. Huang, "Study on optical methods for 3-D shape measurement". Optical Technique, vol. 32, pp. 654-658, 2006.

[4] J. Wang, Y. Zhao, Sh. L. Zhang, et al. "Nano Metrology and Nano Metrology Testing Technology (1)". Aviation Metrology \& Measurement Technology, vol. 15, no. 5, pp. 3-5, 1995.

[5] J. Wang, Y. Zhao, Sh. L. Zhang, et al. "Nano metrology and nano metrology testing technology (2)". Aviation Metrology \& Measurement Technology, vol. 15, no. 5, pp. 3-5, 1995

[6] L. C. Zhang, and J. S. Gao, "Developments of DUV coating technology in CIOM", Optics and Precision Engineering, vol. 20, pp. 2395-2401, 2011.

[7] F. G. Tseng, and J. M. Chang, "Multi-head probe and scanning method thereof", U. S. Patent 2014020140, 2014

[8] D. Nabs, and K. Gensecke, "Device and method for the threedimensional optical measurement of strongly reflective or transpar- ent objects", Available from http://patentscope.wipo.int/sear$\mathrm{ch} / \mathrm{en} /$ detail.jsf?docId $=\mathrm{CN} 84819348 \& \mathrm{recNum}=5 \& \max \mathrm{Rec}=46 \&$ off ice $=\&$ prevFilter $=\&$ sortOption $=$ Pub + Date + Desc \&queryString $=$ ALL NAMES\%3A\%28AFM\%29\&tab=NationalBiblio, 2011

[9] K. N. Joo, "Method and apparatus for performing film thickness measurement using white light scanning interferometry", U. S. Patent 20120218560, 2012

[10] H. Sakai, "Optical measurement device and optical measurement system", WO2014024614, 2014

[11] T. Noda, and H. Deguchi, "Shape measuring apparatus and control method of shape measuring apparatus", U. S. Patent 20140025336, 2014

[12] K. Fukuda, T. Ito, and M. Kobayashi, "Nano indentation of the Resist for Electron Beam Lithography”. JSME annual meeting, vol. 4, pp. 105-106, 2003.

[13] S. Wang, T. B. Xie, and S. P. Chang, "Combined profilometer for ultra-precision surface topography", Optics and Precision Engineering, vol. 19, no.4, pp. 828-835, 2011.

[14] S. Liu, S. Nagasawa, S. Takahashi, and K. Takamasu, "Development of a multi-ball-cantilever AFM for measuring resist surface". Journal of Robotics and Mechatronics, vol. 18, no. 6, pp. 698-704, 2006.

[15] C. Zygo, "New View Accessories", Product guide for the New View 3D Surface Profiler, 2001.

[16] K. Flock, L. Rotter, and M. Arain, "Calibration of an optical metrology system for critical dimension application matching”, Available from http://worldwide.espacenet.com/publicationDetails/biblio? $\mathrm{DB}=$ worldwide.espacenet.com $\& \mathrm{II}=7 \& \mathrm{ND}=3 \&$ adjacent $=$ true $\&$ locale $=$ en_EP $\& F T=$ D\&date $=20131116 \& C C=T W \& N R=201$ 346213A\&KC=A, TW201346213, 2013

(C) Liu et al.; Licensee Bentham Open.

This is an open access article licensed under the terms of the Creative Commons Attribution Non-Commercial License (http://creativecommons.org/licenses/by$\mathrm{nc} / 3.0 /$ ) which permits unrestricted, non-commercial use, distribution and reproduction in any medium, provided the work is properly cited. 